Bryn Mawr College

Scholarship, Research, and Creative Work at Bryn Mawr

College

1972

\title{
Observation of the influence of centrifugal distortion of the methane molecule on nuclear spin relaxation in the gas.
}

Peter A. Beckmann

Bryn Mawr College, pbeckman@brynmawr.edu

M. Bloom

E. E. Burnell

Let us know how access to this document benefits you.

Follow this and additional works at: https://repository.brynmawr.edu/physics_pubs

Part of the Physics Commons

\section{Custom Citation}

P. A. Beckmann, , M. Bloom, and , E. E. Burnell 1972. Observation of the influence of centrifugal distortion of the methane molecule on nuclear spin relaxation in the gas. Canadian Journal of Physics 50.3: 251-258.

This paper is posted at Scholarship, Research, and Creative Work at Bryn Mawr College. https://repository.brynmawr.edu/physics_pubs/139

For more information, please contact repository@brynmawr.edu. 


\section{Canadian \\ Journal of \\ Physics}

Observation of the Influence of Centrifugal

Distortion of the Methane Molecule on

Nuclear Spin Relaxation in the Gas

P. A. BEckMANN, M. Bloom, AND E. E. BuRNELl

Volume $50 \cdot$ Number $3 \cdot 1972$

Pages 251-258 


\title{
Observation of the Influence of Centrifugal Distortion of the Methane Molecule on Nuclear Spin Relaxation in the Gas ${ }^{1}$
}

\author{
P. A. Beckmann, M. Bloom, and E. E. Burnell \\ Department of Physics, University of British Columbia, Vancouver 8, British Columbia \\ Received August 18, 1971
}

\begin{abstract}
The spin-lattice relaxation time $T_{1}$ was measured in gaseous $\mathrm{CH}_{4}$ as a function of density at room temperature between 0.006 and 7.0 amagats. $T_{1}$ was found to pass through a minimum near 0.04 amagats in agreement with previous, less precise measurements. The spin-rotation interaction is the dominant relaxation mechanism in gaseous $\mathrm{CH}_{4}$. Since the spin-rotation constants are accurately known for $\mathrm{CH}_{4}$, the relaxation experiments provide a check on the theory of spin-lattice relaxation for spherical top molecules. In the conventional theory, it is assumed that the correlation function of the spin-rotation interaction is a simple exponential function of time. These experiments show that this assumption is not true for $\mathrm{CH}_{4}$ gas. The observed fine structure in the plot of relaxation rate versus density is attributed to the influence of centrifugal distortion of the $\mathrm{CH}_{4}$ molecule, which removes the degeneracy of rotational states having the same value of the quantum number $J$ by an amount somewhat greater than the nuclear Larmor frequency of $30 \mathrm{MHz}$.
\end{abstract}

Le temps de relaxation spin $\rightarrow$ réseau $T_{1}$ a été mesuré dans le $\mathrm{CH}_{4}$ gazeux, à température ambiante, entre 0.006 et 7.0 amagats. On trouve que $T_{1}$ passe par un minimum au voisinage de 0.04 amagats comme l'indiquaient déjà des mesures antérieures moins précises. L'interaction spin-rotation est le mécanisme dominant de la relaxation dans le $\mathrm{CH}_{4}$ gazeux. Etant donné que les constantes spin-rotation sont connues de façon précise pour le $\mathrm{CH}_{4}$, les expériences de relaxation procurent un moyen de vérifier la théorie de la relaxation spin-réseau pour les molécules assimilables à des toupies sphériques. Dans la théorie conventionnelle, on assume que la fonction corrélation de l'interaction spin-rotation est une simple fonction exponentielle du temps. Ces expériences indiquent que cette hypothèse est fausse pour le $\mathrm{CH}_{4}$ gazeux. La structure fine qui apparaît dans la courbe du taux de relaxation en fonction de la densité est attribué à l'influence de la distortion centrifuge de la molécule de $\mathrm{CH}_{4}$; cette distortion a pour effet de faire disparaître la dégénérescence des états rotationnels ayant une même valeur du nombre quantique $J$ en les séparant par une quantité quelque peu plus élevée que la fréquence nucléaire de Larmor de $30 \mathrm{MHz}$.

Canadian Journal of Physics, 50, 251 (1972)

\section{Introduction}

The nuclear spin-lattice relaxation time $T_{1}$ in low density molecular gases is determined by the nuclear hyperfine interactions (Yi et al. 1968) which couple the nuclear spins to the molecular rotation. Usually, the dominant interaction for nuclei of spin 1/2 is the spin-rotation interaction (Bloom et al. 1967). In order to calculate $T_{1}$ theoretically it is necessary to know not only the spin-rotation coupling constants, but also the correlation functions of the rotational angular momentum $J$ and of certain functions of the molecular orientation (Bloom et al. 1967). These correlation functions have not yet been calculated from first principles for any systems other than $\mathrm{H}_{2}$ gas (Bloom and Oppenheim 1967). The detailed measurements of $T_{1}$ in methane gas as a function of density at room temperature which we report in this paper are of particular theoret-

\footnotetext{
${ }^{1}$ Research supported by the National Research Council of Canada.
}

ical interest at this time. $\mathrm{CH}_{4}$ is the only spherical top molecule for which both spin-rotation coupling constants have been determined with precision using molecular beam techniques ( $\mathrm{Yi}$ et al. 1967, 1971). Therefore, it is possible, on the basis of our measurements, to draw reasonably precise conclusions about molecular reorientation correlation functions.

The strongest conceivable test of the theory of nuclear spin relaxation in low density gases would be to study $T_{1}$ as a function of the nuclear Larmor frequency $\omega_{0}$ over a very wide range of frequencies. If it were possible to tune $\omega_{0}$ through the molecular rotation frequencies, characteristic maxima in the relaxation rate $T_{1}^{-1}$ would occur in complete analogy with the resonant absorption observed in conventional spectroscopy. ${ }^{2}$ The rotational spectrum of $\mathrm{CH}_{4}$

\footnotetext{
${ }^{2}$ As discussed by Hardy (1966), it is actually the difference between the nuclear and molecular Larmor frequencies that is relevant here. In the theoretical expressions of Section 3, $\omega_{0}$ stands for this difference.
} 

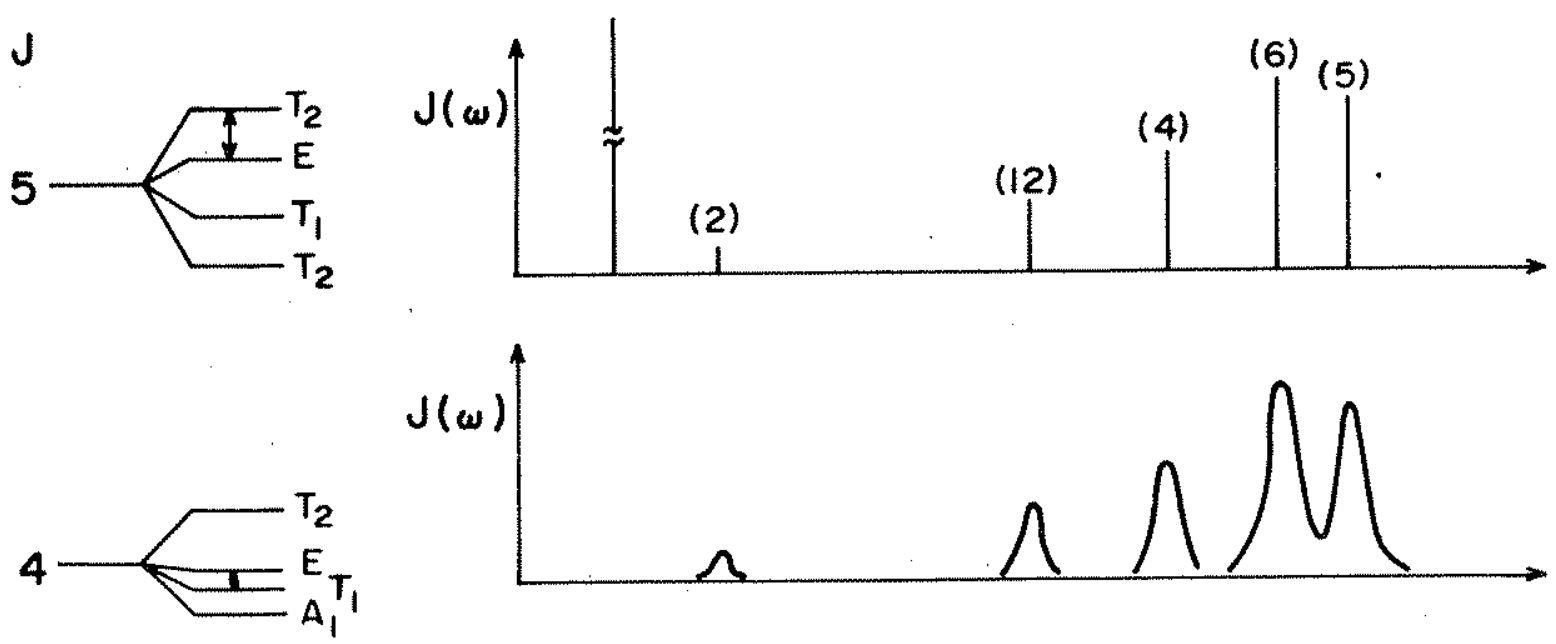

(c)
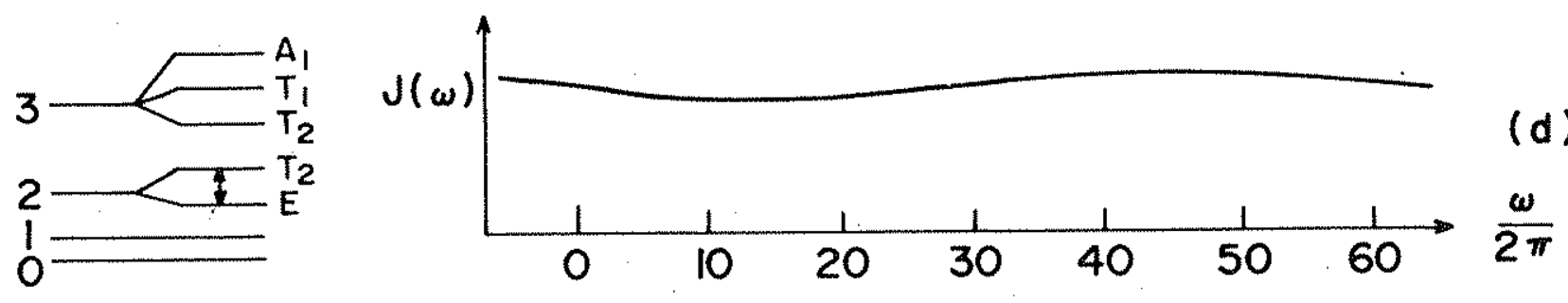

(a)

\section{FREQUENCY (MHz)}

Fig. 1. Schematic diagram illustrating the low-frequency components of the centrifugal-distortion "nuclear spin relaxation rotational spectrum" of $\mathrm{CH}_{4}$ gas at different pressures. (a) Energy level diagram of $\mathrm{CH}_{4}$ molecules in the ground vibrational state for $J \leqslant 5$. The energy leveis are not drawn to scale and are only intended to indicate the classification of the states for each value of $J$ according to the $A(I=2), T(I=1)$, and $E(I=0)$ irreducible representations of the tetrahedral group. The arrows indicate those allowed centrifugal-distortion transitions for the spin-rotation interactions at frequencies less than $60 \mathrm{MHz}$ (Ozier 1971, private communication), which are shown in $(b)$. (b) The low-frequency $(<60 \mathrm{MHz})$ components of the "nuclear spin relaxation free rotational spectrum" for $\mathrm{CH}_{4}$. Contributions are obtained from states $J=2,4,5,6$, and 12 according to the energy levels published by Hecht (1960). The $\omega_{k}=0$ terms are very much larger than any of the $\omega_{k} \neq 0$ terms. (c) The spectrum of $(b)$ slightly broadened by collisions $\left(\omega_{k} \tau_{c} \gg 1\right)$. The $\omega_{k}=0$ terms are not included. (d) The spectrum of $(b)$ greatly broadened by collisions $\left(\omega_{k} \tau_{c} \ll 1\right)$. The $\omega_{k}=0$ terms are included.

is illustrated in Fig. 1 where the $\Delta J \neq 0$ transitions have not been included because they are of much too high frequency to contribute to the relaxation of a dilute gas. Such an observation of the rotational spectrum using nuclear spin relaxation has not been considered because $\omega_{0}$ is limited by the available laboratory magnetic fields to the radio-frequency regime while typical molecular rotational frequencies lie in the infrared. Measurements of $T_{1}$ in dilute gases are usually interpreted in a manner similar to "nonresonant absorption" measurements which are familiar to those who study dielectric relaxation (Birnbaum 1967). When $T_{1}^{-1}$ is plotted as a function of density at a fixed value of $\omega_{0}$, a characteristic maximum occurs near the density at which the correlation time for molecular reorientation is of the order of $\omega_{0}^{-1}$. The detailed information available from measurements of the $T_{1}$ minimum (or $T_{1}^{-1}$ maximum) was first demonstrated in a study of $\mathrm{H}_{2}$ gas by Hardy (1966).

Spherical top molecules such as $\mathrm{CH}_{4}$ are systems in which it should be possible to do the type of "spin-lattice relaxation spectroscopy" mentioned previously. The reason for this is that in spherical top molecules the centrifugal distortion energy partially removes the degeneracy associated with the quantum number $J$. For the case of $\mathrm{CH}_{4}$ it is well known that these centrifugal distortion splittings range from several $\mathrm{MHz}$ to hundreds of $\mathrm{MHz}$ over the rotational states which are appreciably populated at room temperature (Hecht 1960). In fact, the $8 \mathrm{MHz}$ splitting of the $J=2$ state has recently been measured directly by Ozier et al. (1970). 
The spin-rotation interaction in spherical top molecules is characterized by a scalar (isotropic) term having coupling constant $C_{\mathrm{a}}$ and a tensor (anisotropic) term having coupling constant $C_{\mathrm{d}}$ (Anderson and Ramsey 1966). The rotational states which are split by the centrifugal distortion interaction can be coupled in general by the tensor part of the spin-rotation interaction. Therefore, the characteristic effects of the centrifugal distortion splittings should manifest themselves in a plot of $T_{1}^{-1}$ versus density in the density range where the collision frequency is of the same order as the average distortion splitting. In a preliminary study of the $T_{1}$ minimum in dilute $\mathrm{CH}_{4}$ gas, Dong and Bloom (1970) failed to observe these effects, though the magnitude of $C_{\mathrm{d}}$ suggests that they should be observable. We have repeated the experiments of Dong and Bloom on $\mathrm{CH}_{4}$ and, with improved precision and care taken to eliminate small systematic errors, have observed the effects of the centrifugal distortion splittings.

\section{Experimental Methods}

The measurements of $T_{1}$ were carried out at $30 \mathrm{MHz}$ using pulse techniques. The apparatus used by Dong and Bloom (1970) was modified to give an improved signal-to-noise ratio. This made possible the measurement of $T_{1}$ at densities as low as 0.006 amagats, almost 3 times lower than that attained by Dong and Bloom. All the measurements were taken at room temperature $(295 \mathrm{~K})$.

The modifications to the spectrometer described by Dong and Bloom (1970) are as follows.

1. Sample cell. The sample cell was a brass bomb with a Kovar feed-through for the RF. The coil was approximately $4 \mathrm{~cm}$ long and $2 \mathrm{~cm}$ in diameter and consisted of 12 turns of No. 14 copper wire which had been stripped and cleaned. The coil fitted tightly inside a glass tube and the high voltage end was insulated by Torr seal epoxy from the brass to prevent dielectric breakdown at low densities.

2. Matching circuits. The tuning capacitor was placed as close as possible to the sample coil just outside the sample cell. The parallel-tuned circuit was coupled to the transmitter via a $4.7 \mathrm{pf}$ capacitor and a $1 / 2$ wavelength line, and to the preamplifier via a $4.7 \mathrm{pf}$ capacitor and two
$1 / 4$ wavelength lines in series with cross diodes to ground between them.

3. Signal detection. A fast-recovery preamplifier was constructed following a design by Riehl (1966). This made possible measurements using the "boxcar integrator" approximately $10 \mu \mathrm{s}$ after the RF pulse. The use of a fast-recovery amplifier is important at low densities since the spin-spin relaxation time becomes shorter as the density is decreased (Hardy 1966).

Most of the measurements were made by first inverting the nuclear magnetization with an RF pulse, usually called a " $\pi$ pulse", and then sampling the recovery of the nuclear magnetization following a " $\pi / 2$ pulse" using a boxcar integrator. One important problem involved the long term recovery of the amplifiers after a pulse. Thus even in the absence of a sample an apparent "signal" is observed which changes with the $\pi / 2-\pi$ pulse spacing. It was very important at all densities to subtract a "vacuum signal" from the signal measured in the presence of the gas in order to avoid a systematic error. A detailed description of the experimental technique has been given by Beckmann (1971).

The experimental results are presented in Section 4. In assessing the reliability of the results one should treat the measurements at the lowest densities with caution. At densities above $\sim 0.02$ amagats all estimates of standard deviations in $T_{1}^{-1}$ from individual measurements yielded errors less than the spread in values obtained from the best fits. Thus the random errors as illustrated by the scatter of the data in Fig. 2, for example, are associated in some sense with the long term stability of the apparatus. At the lower densities, the scatter of the results is of the same order as the uncertainty in the individual measurements. A detailed discussion of the treatment of the data can be found elsewhere (Beckmann 1971).

\section{Review of the Theory}

Nuclear spin relaxation in spherical top molecules due to the spin-rotation interaction has been investigated quite thoroughly and we give here only a brief review with emphasis on the physical processes involved. Hubbard (1963) and Blicharski (1963) arrived at expressions for $T_{1}$ in liquids composed of spherical top molecules. Bloom et al. (1967) extended the theory to 


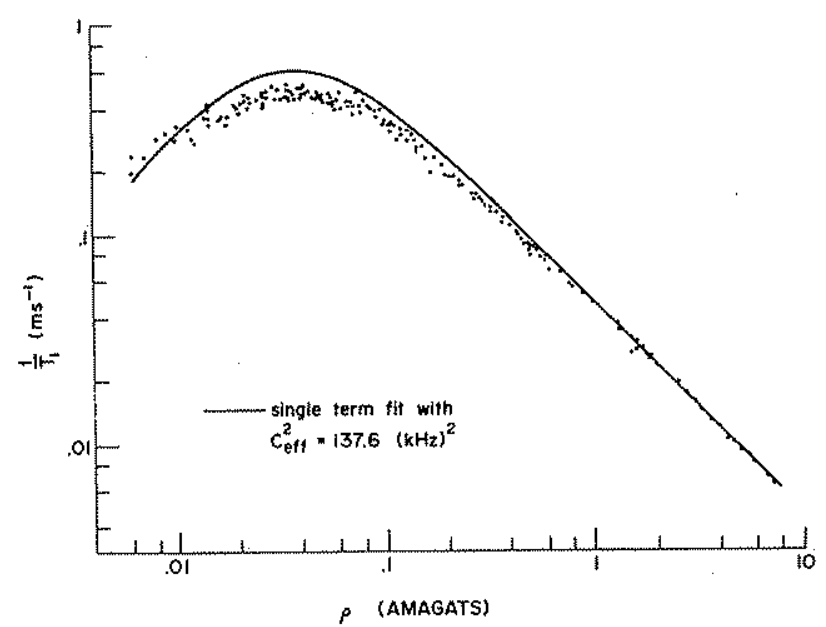

FIG. 2. Plot of $1 / T_{1}$ as a function of the density of methane gas at $295 \mathrm{~K}$. The theoretical curve is given by [10] with the effective spin-rotation coupling constant determined from molecular beam measurements.

gases composed of symmetric and spherical top molecules. These papers use the general theory of nuclear spin relaxation as described in the classic text of Abragam (1961) as a starting point. McCourt and Hess $(1970)^{3}$ have shown how to incorporate the theory of nuclear spin relaxation in polyatomic molecules into the Boltzmannequation approach developed by Chen and Snider (1967, 1968, 1969).

The spin-lattice relaxation rate $T_{1}^{-1}$ in a dilute molecular gas may be written in the following form (Bloom et al. 1967). ${ }^{2}$

$$
\frac{1}{T_{1}}=a J\left(\omega_{0}\right)=a \sum_{k} G_{k} j_{k}\left(\omega_{0}-\omega_{k}\right)
$$

where $a$ is a constant; $G(t)$, the correlation function of the rotational operators in the spinrotation interaction, has been expressed in the form

$$
\text { [2] } \quad G(t)=\sum_{k} G_{k}(t)=\operatorname{Re} \sum_{k} G_{k} \mathrm{e}^{i \omega_{k} t} g_{k}(t)
$$

and

$$
j_{k}(\omega)=\int_{-\infty}^{\infty} \mathrm{e}^{i \omega t} g_{k}(t) \mathrm{d} t
$$

In eqs. $1-3$ the index $k$ labels a particular pair of rotational states between which the spinrotation interaction has nonzero matrix elements and $\hbar \omega_{k}$ is the energy difference between these states. The quantity $G_{k}$ is proportional to the

\footnotetext{
${ }^{3}$ See also the short notes by Ozier (1971) and McCourt and Hess (1971).
}

square of the matrix element of the spin-rotation interaction between these states and to the Boltzmann factor of one of the states. The spectral density $J(\omega)$ is the rotational spectrum illustrated in Fig. 1. In the absence of collisions, the reduced correlation function $g_{k}(t)=g_{k}(0)=1$ in which case the spectrum consists of a superposition of $\delta$ functions at the frequencies $\omega_{k}$ having intensities $G_{k}$ (Fig. $1 b$ ). This spectrum could be termed the "nuclear spin relaxation free rotational spectrum." Explicit expressions for this spectrum have been given by Bloom et al. (1967) neglecting nuclear spin symmetry and centrifugal distortion effects. Under these conditions the spectrum for spherical top molecules may be classified conveniently in two categories corresponding to the selection rules $\Delta J=0$ and $\Delta J \neq 0$ respectively. Since the $\Delta J \neq 0$ terms contribute only in the infrared region of the spectrum for a dilute gas, they may be completely ignored in calculating $T_{1}$. The $\Delta J=0$ terms would be peaked at zero frequency.

When the permutation symmetry of the four protons in $\mathrm{CH}_{4}$ is taken into account, the centrifugal distortion energy partially removes the degeneracy of the states characterized by the $J$ quantum number (Hecht 1960). (More accurate values of the centrifugal distortion splittings are now available (Husson and Dang Nhu 1971).) It may be shown that the nondegenerate states of a $J$ manifold may be classified according to the irreducible representations of the tetrahedral group. The allowed states are shown schematically for $J \leqslant 5$ for $\mathrm{CH}_{4}$ in Fig. $1 a$. In the case of $\mathrm{CH}_{4}$ there is a one-to-one correspondence between the total nuclear spin per molecule $I$ and the irreducible representations. The meta $(A)$, ortho $(T)$, and para $(E)$ spin symmetry species have $I=2,1$, and 0 respectively.

Since the spin-rotation interaction is linear in the nuclear spin operators, the only nonzero matrix elements satisfy the selection rule $\Delta I=0$, \pm 1 . Therefore, in addition to a large free rotational term at $\omega_{k}=0$ corresponding to matrix elements of the spin-rotation interaction between states $A \leftrightarrow A$ and $T \leftrightarrow T$, there exist terms at $\omega_{k} \neq 0$ corresponding to certain nonzero $A \leftrightarrow T, T \leftrightarrow E$, and $T \leftrightarrow T$ matrix elements (Ozier 1971). The frequencies of all the $\omega_{k} \neq 0$ terms in the spectrum below $60 \mathrm{MHz}$ are shown in Fig. $1 b$ according to energy levels given by Hecht (1960). Although we have not calculated 
the matrix elements of the spin-rotation interaction for these transitions, the theory which neglects nuclear spin symmetry indicates that the square of the matrix elements should be proportional to $J(J+1)$ for $J \gg 1$. We have therefore indicated the "relative intensities" of the different lines in the spectrum of Fig. $1 b$ by the product of $J(J+1)$ and $(2 J+1) \exp [-B J(J$ $+1) k T$ ], where $B$ is the rotational constant. None of the intensities of the $\omega_{k} \neq 0$ lines in Fig. $1 b$ is more than a few percent of the $\omega_{k}=0$ intensity.

It is clear that nuclear spin relaxation cannot take place in the absence of collisions between the molecules. The effect of collisions at low densities is to broaden the free molecule rotational lines as indicated schematically in Figs. $1 c$ and $1 d$ though at higher densities the effect of collisions is much more complex (see e.g. BenReuven 1966; Alekseyev et al. 1968). We shall assume (Dong and Bloom 1970) that the rotational lines are Lorentzian at low densities, i.e.

$$
j_{k}(\omega)=\frac{2 \tau_{k}}{1+\omega^{2} \tau_{k}^{2}}
$$

where the correlation time $\tau_{k}$ is defined by

$$
g_{k}(t)=\exp \left(-t / \tau_{k}\right)
$$

From the properties of the spin-rotation interaction (Anderson and Ramsey 1966) we know that the scalar term (of amplitude $C_{\mathrm{a}}$ ) involves only $\omega_{k}=0$ terms while the tensor term (of amplitude $C_{\mathrm{d}}$ ) does connect states whose energies differ due to the centrifugal distortion energy (Anderson and Ramsey 1966; Ozier et al. 1970). A simple extrapolation of the theory of Bloom et al. (1967) then gives the following general expression for $T_{1}$ which should be valid at high temperatures.

$$
\text { [6] } \begin{aligned}
\frac{1}{T_{1}}=\frac{4 \pi^{2}}{\alpha}\left[C_{\mathrm{a}}^{2} \frac{\tau_{1}}{1+\omega_{0}^{2} \tau_{1}^{2}}\right. & +\frac{4}{45} C_{\mathrm{d}}^{2} \\
& \left.\times \sum_{k} \frac{F_{k} \tau_{k}}{1+\left(\omega_{0}-\omega_{k}\right)^{2} \tau_{k}^{2}}\right]
\end{aligned}
$$

where $\alpha=\hbar^{2} /\left(2 I_{0} k T\right), I_{0}$ being the moment of inertia of $\mathrm{CH}_{4}$, and $F_{k}$ is a measure of the sum of the squares of the matrix elements of the tensor part of the spin-rotation interaction between states having energy differences $\hbar \omega_{k}$. The $F_{k}$ satisfy the normalization condition

$$
\sum_{k} F_{k}=1
$$

At the present time the values of $F_{k}$ have not been computed explicitly though the procedure for doing so is implicit in Ozier et al. (1968). A proper calculation of the $F_{k}$ involves taking into account nuclear spin symmetry and the conversion between nuclear spin symmetry species (Curl et al. 1967; Ozier and Yi 1967) as we shall discuss in the last chapter of this paper. In order to discuss the significance of our experimental results we shall find it convenient to introduce some limiting cases for [6]. Before doing this we note that at low densities, the correlation times are inversely proportional to the density at constant temperature so that

$$
\frac{\rho_{k}}{\left|\omega_{0}-\omega_{k}\right|} \frac{1}{\rho}=\tau_{k}=\frac{1}{\left\langle\sigma_{k} v\right\rangle \rho}
$$

where $\sigma_{k}$ is an apparent cross section for the $k$ th rotational line, and $\langle>$ denotes an average over a Maxwell-Boltzmann velocity distribution. The constant $\rho_{k}$ is the density at which

$$
\left|\omega_{0}-\omega_{k}\right| \tau_{k}=1 \quad\left(\text { defines } \rho_{k}\right. \text { ) }
$$

i.e. $\rho_{k}$ is the density at which the $k$ th term in [6] makes its maximum contribution to the relaxation rate.

Case 1. If the centrifugal splitting is negligible and $\tau_{k}=\tau_{1}$ for all $k$, eq. 6 reduces to

where

$$
\frac{1}{T_{1}} \approx \frac{4 \pi^{2} C_{\mathrm{eff}}^{2}}{\alpha \omega_{0}} \frac{\rho_{1} / \rho}{1+\rho_{1}^{2} / \rho^{2}}
$$

This was the approximation used by Dong and Bloom (1970) to interpret their data.

Case 2. If the centrifugal distortion spectrum is replaced by a single "average" line at frequency $\Omega$ and the scalar and tensor terms have correlation times $\tau_{1}$ and $\tau_{1}^{\prime}$ with associated characteristic densities $\rho_{1}$ and $\rho_{1}^{\prime}$ respectively,

$$
\text { [12] } \begin{aligned}
\frac{1}{T_{1}} \approx \frac{4 \pi^{2}}{\alpha}\left[\frac{C_{\mathrm{a}}^{2} \frac{\rho_{1} / \rho}{\omega_{0}} \frac{\rho_{1}^{2} / \rho^{2}}{1+\rho^{2}}}{\left.+\frac{4}{45} \frac{C_{\mathrm{d}}^{2}}{\Omega} \frac{\rho_{1}^{\prime} / \rho}{1+\left(\rho_{1}^{\prime}\right)^{2} / \rho^{2}}\right]}\right.
\end{aligned}
$$

where $\Omega=\left|\omega_{0}-\omega_{k}\right|$ for a single "average" $k$. 


\section{Experimental Results and Discussion}

The experimental results shown in Fig. 2 are the relaxation rate $T_{1}^{-1}$ as a function of density $\rho$. The dimensionless unit amagats is the ratio of the density under consideration to the density at standard temperature and pressure. It was found that the data could not be adequately fit by the approximations of Case 1, i.e. a single correlation time and no centrifugal distortion as expressed by [10]. Figure 2 shows an example of a fit in which the spin-rotation coupling constants $C_{\mathrm{a}}=10.4 \mathrm{kHz}$ and $C_{\mathrm{d}}=18.2 \mathrm{kHz}$, as determined from molecular beam measurements (Yi et al. 1967), have been used to fix $C_{\text {eff }}^{2}=137.6(\mathrm{kHz})^{2}$ in [10]. The constant $\rho_{1}$ was then determined from $T_{1} / \rho$ at densities above 1 amagat. The experimental result of

$$
\begin{aligned}
& T_{1} / \rho=21.9 \pm 0.4 \mathrm{~ms} / \text { amagat } \\
& \qquad \rho>1 \text { amagat }
\end{aligned}
$$

yields a value of $\rho_{1}=0.039$ amagats where the error quoted corresponds to one standard deviation. In the paper of Dong and Bloom (1970) they reported a value of $T_{1} / \rho$ of $20.0 \mathrm{~ms} /$ amagat and that the dependence of $T_{1}$ on $\rho$ did fit [10]. The disagreement between our results and those obtained by Dong and Bloom (1970) is discussed in Section 5.

Having eliminated [10] as a reasonable fit of the data, we now turn to [12] and examine the special case of $\Omega=\omega_{0}$ (no centrifugal distortion splitting) but $\rho_{1} \neq \rho_{1}^{\prime}$, i.e. we allow the tensor term to have a different correlation time than the scalar term. Rather than randomly picking values for the two parameters $\rho_{1}$ and $\rho_{1}^{\prime}$ we use the experimental result that for $\rho>1$ amagat, $T_{1} \propto \rho$ with a constant of proportionality given by [13]. Assuming that $\rho_{1}, \rho_{1}^{\prime} \ll 1$ amagat, [12] and [13] give a linear relationship between $\rho_{1}$ and $\rho_{1}^{\prime} . T_{1}^{-1}$ was then plotted as a function of $\rho$ for different choices of the remaining parameter $\rho_{1}^{\prime}$. It proved to be impossible to fit the data near the maximum in $T_{1}^{-1}$ by this procedure. In fact the resulting theoretical curves were in no case much different from that shown in Fig. 2.

Since it is known that the centrifugal distortion splittings are by no means negligible as compared with $\omega_{0}$ (Hecht 1960), the conclusion that they must be taken into account in interpreting our $T_{1}$ measurements is not at all surprising. The influence of $\Omega \neq \omega_{0}$ has been taken into account using the approximate expression [12] with $\rho_{1}, \rho_{1}^{\prime}$, and $\Omega$ treated as parameters with which to fit the experimental data.

We do have information about the magnitude of the centrifugal distortion splittings in the different rotational states (Hecht 1960). We have shown in Fig. 1 all of the relevant rotational splittings below $60 \mathrm{MHz}$. However, most of the intensity of the centrifugal distortion spectrum lies between $60 \mathrm{MHz}$ and several hundred $\mathrm{MHz}$. On the basis of this information and the assumption that the apparent cross section for molecular reorientation is not too different for the different rotational lines (i.e. $\tau_{1} \approx \tau_{1}^{\prime}$ ) it seems reasonable to invoke the following assumptions

$$
\rho_{1}^{2} \ll\left(\rho_{1}^{\prime}\right)^{2} \ll(1 \text { amagat })^{2}
$$

This is equivalent to saying that $\Omega \gg \omega_{0}$, but much smaller than the collision frequency at a pressure of about $1 \mathrm{~atm}$ at room temperature. The use of approximation [14] together with [12] and [13] is sufficient to determine the following parameters.

$$
\rho_{1}=0.039 \text { amagats } ; \quad \rho_{1}^{\prime} / \Omega=2.2 \times 10^{-10}
$$

\section{amagats}

Furthermore, [14] implies that the $C_{\mathrm{a}}$ contribution to $T_{1}^{-1}$ in [12] should give a reasonable fit of $T_{1}^{-1}$ near its maximum. This expectation is indeed borne out remarkably well as shown in Fig. 3. Having accounted for the $C_{\mathrm{a}}$ contribution to the relaxation rate, we now plot $\left[T_{1}^{-1}\right]_{C_{d}}$ versus $\rho$ and attempt to interpret it in terms of

$$
\begin{aligned}
{\left[\frac{1}{T_{1}}\right]_{C_{a}} } & =\left[\frac{1}{T_{1}}\right]_{\text {expt }}-\left[\frac{1}{T_{1}}\right]_{C_{\mathrm{a}}} \\
& =\frac{16 \pi^{2} C_{d}^{2}}{45 \alpha \Omega}\left[\frac{\rho_{1}^{\prime} / \rho}{1+\left(\rho_{1}^{\prime}\right)^{2} / \rho^{2}}\right]
\end{aligned}
$$

for different values of $\Omega$ using $\rho_{1}^{\prime}=2.2 \times 10^{-10}$ $\Omega$ amagats. This is done in Fig. 4 for 3 values of $\Omega$. A reasonable fit of the experimental results is obtained for $5 \omega_{0} \lesssim \Omega \lesssim 8 \omega_{0}$ which implies that the approximate expression $T_{1}^{-1}$ in [12] is consistent with an average centrifugal distortion frequency of about $200 \mathrm{MHz}$, which is quite close to the average distortion frequency at room temperature. Finally, [12] is plotted in Fig. 3 for $\Omega=6 \omega_{0}$. Grood agreement with experiment is obtained except for $\rho<0.01$ amagats. 


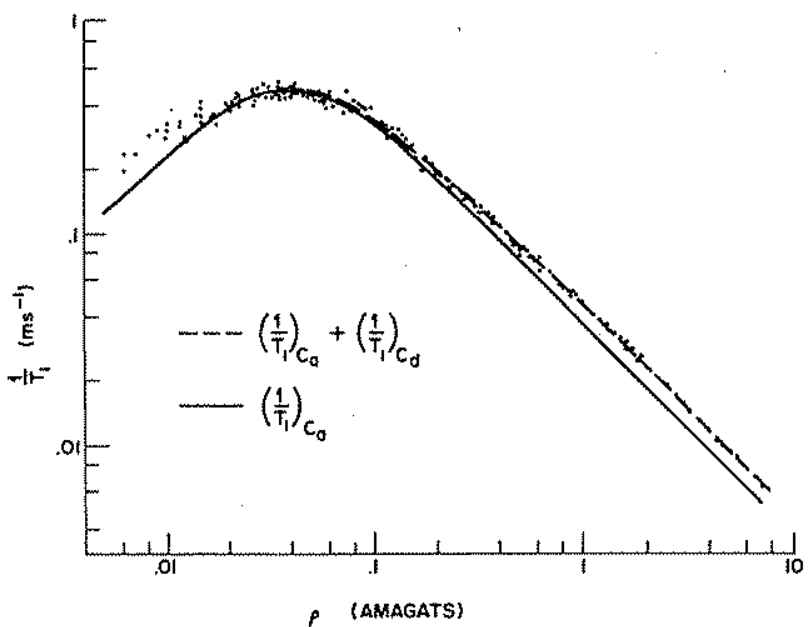

Frg. 3. Plot of $1 / T_{1}$ as a function of the density of methane gas at $295 \mathrm{~K}$. The solid curve is the theoretical contribution of the scalar $\left(C_{\mathrm{a}}\right)$ part of the spin-rotation interaction to the relaxation rate using the correlation time derived from the theoretical curve of Fig. 2. The dashed curve includes a contribution due to the tensor term $\left(C_{\mathrm{d}}\right)$ as described in the text. (The difference of the 2 curves is indicated by the $\Omega=6 \omega_{0}$ curve in Fig. 4.)

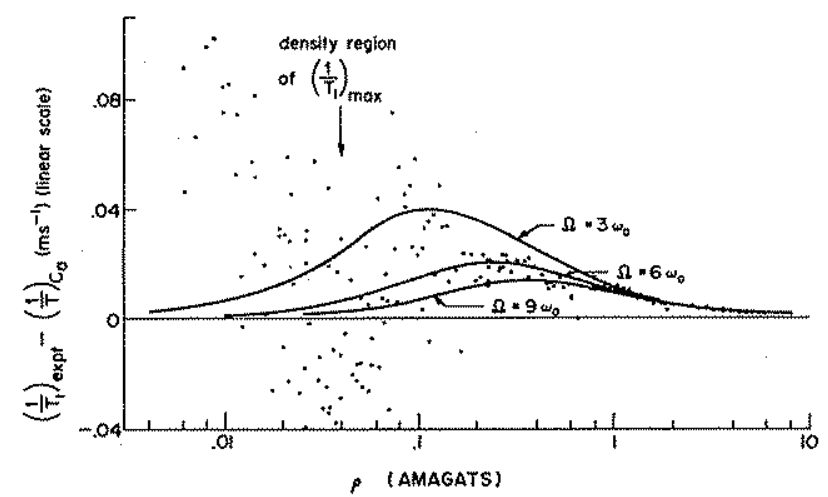

FiG. 4. Comparison of the density dependence of the contribution of the tensor part of the spin-rotation interaction with the simplified model of [12] for different values of the "average centrifugal splitting" $\Omega$.

\section{Discussion on Disagreement with Previous Results}

The report by Dong and Bloom (1970) that the dependence of $T_{1}$ on $\rho$ did fit [10] was based on 11 values of $T_{1}$ whereas we could not fit the 185 data points shown in Fig. 2 to [10]. As well as the many more data points we report, our signal to noise was better than that of Dong and Bloom (1970) so our study represents a more critical test of [10].

Dong and Bloom (1970) report that $T_{1} / \rho$ is constant for $\rho>0.25$ amagats and, from data at 4 values of the density between 0.25 and 1.2 amagats, they have determined $T_{1} / \rho=20.0$ ms/amagat. We report that $T_{1} / \rho$ does not become constant until $\rho>1.0$ amagat and, then, our value of $T_{1} / \rho$ is $10 \%$ higher than theirs. We agree with the density value at which the $T_{1}^{-1}$ maximum occurs, but our value of $T_{1}^{-1}$ at the $T_{1}^{-1}$ maximum is about $25 \%$ lower than theirs. The results of Dong and Bloom (1970) are in agreement with those reported by Lalita and Bloom (1971). Both groups used essentially the same apparatus and in particular, the same sample chamber. Gerritsma et al. (1971) have reported measurements of $T_{1}=2.38 \mathrm{~s}$ at 108 ama- gats and $T_{1}=2.79 \mathrm{~s}$ at 126 amagats at a temperature of $299.2 \mathrm{~K}$. These measurements give $T_{1} / \rho$ values in agreement with our value of $21.9 \pm 0.4$ ms/amagat. The samples of Gerritsma et al. (1971) were contained in sealed glass or quartz tubes. We extended the measurements of $T_{1}$ with our sample chamber (see Section 2) from 7 amagats (which is the highest density reported in this paper) to 17 amagats and observed no change in our value of $T_{1} / \rho$. (Seventeen amagats is the highest density at room temperature that the sample chamber discussed in Section 2 can withstand.)

It seems likely that the results obtained with the sample chamber of Dong and Bloom (1970) and Lalita and Bloom (1971) are influenced at higher densities by a small $(\lesssim 10 \%)$ contribution to the relaxation rate due to surface effects. (A lower value of $T_{1} / \rho$ corresponds to a faster relaxation rate.) Work is in progress to establish whether or not the lower $T_{1} / \rho$ value reported by Dong and Bloom (1970) and Lalita and Bloom (1971) is really due to wall relaxation, but it is doubtful that such effects could influence our results by more than a few percent for $\rho \gtrsim 0.01$ amagats. The qualitative conclusions we have drawn in Section 4 on the importance of the centrifugal distortion splittings would not be affected by such small effects.

\section{Concluding Remarks}

There exists no theory of nuclear spin relaxation in gases of polyatomic molecules which include the effects of nuclear spin symmetry. However, it is clear that for tetrahedral molecules such as $\mathrm{CH}_{4}$, the partial removal of the degeneracy of the rotational states by the centrifugal-distortion energy should give rise to a characteristic " $T_{1}$ spectrum" in the RF range. The experimental results reported in this paper 
are in agreement at densities $\rho>0.01$ amagats with the predictions of a simple model in which the centrifugal-distortion $T_{1}$ spectrum is replaced by a single line at an average frequency $\Omega$. According to our results, it should be possible to measure the centrifugal-distortion $T_{1}$ spectrum directly by making precise measurements at a low density of $T_{1}$ as a function of frequency in the range $10 \mathrm{MHz} \lesssim \omega / 2 \pi \lesssim 200 \mathrm{MHz}$, which corresponds to an easily accessible range of magnetic fields below $5 \times 10^{4} \mathrm{G}$.

Our experimental values of $T_{1}^{-1}$ below 0.01 amagats are somewhat higher than predicted by the simple "average frequency" model. This is the type of effect anticipated for terms in the $T_{1}$ spectrum which are approximately tuned to the nuclear Larmor frequency, such as the $J=12$ line near $30 \mathrm{MHz}$ and the $J=4$ line near $40 \mathrm{MHz}$ which are illustrated in Fig. 1. At very low densities the contributions to $T_{1}^{-1}$ of a term tuned to the Larmor frequency should be proportional to $\rho^{-1}$ while all other terms should give contributions proportional to $\rho$. Thus, the terms near $30 \mathrm{MHz}$ could account for the discrepancy of about $30 \%$ between theory and experiment at $\rho=0.006$ amagats. In the same way, wall relaxation could give rise to this discrepancy without seriously affecting the results at higher densities. An order of magnitude calculation indicates that the centrifugal-distortion terms near 30 and $40 \mathrm{MHz}$ could account for the discrepancy, but a more rigorous theory which involves nuclear spin symmetry effects is required to make a definitive interpretation of the low density results. Measurements at a frequency different from $30 \mathrm{MHz}$ could also settle this question.

The spin-lattice relaxation rate in $\mathrm{CH}_{4}$ gas at low densities is closely related to the rate of conversion between nuclear spin symmetry species. The influence of the centrifugal-distortion splittings on the density dependence of the conversion rate in $\mathrm{CH}_{4}$ has, in fact, been discussed explicitly by Ozier and Yi (1967). A rough measure of the conversion rate is given by the contribution of the $C_{\mathrm{d}}$ terms to $T_{\mathrm{l}}^{-1}$ which may be obtained from the analysis of our results given in Section 4. It may be seen from Fig. 4 that our derived results for $\left[T_{1}^{-1}\right]_{C_{\mathrm{d}}}$ are of the same order of magnitude as the estimated conversion rate of $10 \mathrm{~s}^{-1}$ at 10 Torr (0.013 amagats) given by Ozier and Yi (1967).

\section{Acknowledgments}

We wish to thank Dr. K. Lalita for her help in the experiments and Dr. Irving Ozier for many helpful discussions. One of us (M.B.) wishes to express his appreciation of some stimulating remarks made by the anonymous referee of the paper by Dong and Bloom (1970) which led to the experimental study whose results are reported in this paper. One of us (E.E.B.) thanks the National Research Council of Canada for the award of a Postdoctorate Fellowship.

AbraGam, A. 1961. The principles of nuclear magnetism (Oxford University Press, London).

Alekseyey, V., Grasiuk, A., Ragulsky, V., Sobelman, I., and FAIZALOV, F. 1968. IEEE J. Quant. El. 4, 654.

Anderson, C. H. and Ramsey, N. F. 1966. Phys. Rev. $149,14$.

BeckmanN, P. A. 1971. M.Sc. Thesis, University of British Columbia, Vancouver, British Columbia, (Unpublished).

Birnbaum, G. 1967. Advances in chem. phys. Vol. 12, edited by J. Hirschfelder (Wiley and Sons, Inc., New York), pp. 487-548.

Blicharski, J. S. 1963. Acta Phys. Polon. 24, 817.

Ben-Reuven, A. 1966. Phys. Rev, 145, 7.

Bloom, M., Bridges, F. D., and Hardy, W. N. 1967. Can. J. Phys. 45, 3533.

Bloom, M. and Oppenheim, I. 1967. Advances in chem. phys. Vol. 12, edited by J. Hirschfelder (Wiley and Sons, Inc., New York), pp. 549-599.

Chen, F. M. and SNider, R. F. 1967. J. Chem. Phys. 46, 3937. 1968. J. Chem. Phys. 48, 3185.

1969. J. Chem. Phys. 50, 4082.

Curl, R. F., JR., Kaspar, J. V. V., and PITzer, K. S. 1967. J. Chem. Phys. 46, 3220.

Dong, R. Y. and Bloom, M. 1970. Can. J. Phys. 48, 793.

Gerritsma, C. J., Oosting, P. H., and Trappeniers, N. J. 1971. Physica, 51, 381.

Hardy, W. N. 1966. Can. J. Phys. 44, 265.

Hecht, K. T. 1960. J. Mol. Spectrosc. 5, 355, 390.

Hubbard, P. S. 1963. Phys. Rev. 131, 1155.

Husson, N. and Dang Nru, M. 1971. J. Phys. 32, 627.

Lalita, K. and BloOM, M. 1971. Chem. Phys. Lett. 8, 285.

MCCourt, F. R. and Hess, S. 1970. Z. Naturforsch. 25a, 1169.

1971. Z. Naturforsch. 26a, 1234.

OzIER, I. 1971. Z. Naturforsch. 26a, 1232.

OZIER, I. and YI, P. 1967. J. Chem. Phys. 47, 5458.

OzIer, I., Crapo, L. M., and Lee, S. S. 1968. Phys. Rev. $172,63$.

Ozier, I., Yl, P., Khosla, A., and Ramsey, N. F. 1970. Phys. Rev, Lett. 24, 642.

RieHL, J. W. 1966. Ph.D. Thesis, Massachusetts Institute of Technology, Cambridge, Mass. (Unpublished).

Yi, P., Ozier, I., Khosla, A., and RamSey, N. F. 1967. Bull. Am. Phys. Soc. 12, 509.

Yı, P., Ozier, I., and Anderson, C. H. 1968. Phys. Rev, 165,92 .

Yi, P., Ozier, I., and Ramsey, N. F. 1971. J. Chem. Phys. To be published. 